\title{
Sobre la posibilidad de un conocimiento autónomo de la educación y su aplicación a finalidades educativas
}

\author{
FERNANDO GIL CANTERO \\ Universidad Complutense de Madrid
}

\begin{abstract}
SUMMARY.-The aim of this paper is to analyce the posibility of an autonomous knowledge of education as a epistemological differenciating criterion in pedagogy. The problem arises when deffending such an autonomous knowledge involves the rejection of other ways of understanding education because they do not promote that autonomy. The mistake lies in identifying epistemological differenciating criterions with what is valuable in order to educate.

This work try to show that the understanding or the theory of Education as philosophical theories provides a valuable knowledge to education, as well as that knowledge is necessary in order to reach the autonomy above mentioned. It occurs the same with the understanding of the theory of education as applied theories. Each case is analyzed through the knowledge of the educational aims.
\end{abstract}

\section{INTRODUCCIÓN}

Actualmente, se tiende a considerar que la autonomía o sustantividad del conocimiento de la educación es un logro histórico al que antes no se atendía y ahora es la razón básica para validar o descalificar pedagógicamente múltiples aportaciones (cf. Touriñán, 1987). Esta imprescindible y acertada visión tiene, sin embargo, el riesgo de llegar a identificar valor pedagógico y autonomía científica. Nuestro propósito es mostrar que esa identificación no puede darse siempre y que, en consecuencia, hay una serie de límites que caracterizan muy peculiarmente —en ningún caso deslegitiman - la aspiración a un conocimiento autónomo de la educación.

\section{GENERALIZACIONES EPISTEMOLÓGICAS Y CONOCIMIENTO AUTÓNOMO}

Simplificando, tal vez en exceso, podríamos decir que la educación genera dos tipos de discursos. Por un lado, tenemos un discurso referido a los elementos de la acción educativa. Por otro, cuando de lo que se trata no es de partes específicas de esa acción, sino de explicaciones epistemológicas, entonces, lo que tenemos son generalizaciones sobre el modo en que se ha de conocer la educación, y cómo se debe alcanzar el conocimiento educativo de algo. En estos casos, se difunden metanor- 
mas de conocimiento, visiones que caracterizan además la identidad intelectual y profesional del pedagogo.

Dos son las características que nos interesa destacar del último tipo de discurso. En primer lugar, las generalizaciones epistemológicas son las que determinan qué es lo que puede llegar a ser un elemento de la acción educativa y cuál es el sentido pedagógico de esa acción considerada en su totalidad. En segundo lugar, el crecimiento del conocimiento pedagógico no se establece necesariamente añadiendo nuevos elementos de estudio o de práctica, sino incrementando el sentido entre los elementos ya existentes o respecto a las nuevas adquisiciones, pero siempre en función de la validación que proporcione el paradigma en vigor de las generalizaciones epistemológicas sobre el conocimiento de la educación.

Así, la consideración de la educación como una teoría científico tecnológica, no expresa necesariamente ni que los elementos más importantes de la acción educativa sean aquellos que consideremos más «técnicos» o «tecnológicos» (materiales, procesos, medios, etc.), ni tampoco que la aceptación de nuevos elementos o teorías provinientes de otros campos o ciencias, tenga que depender de su rango tecnológico. Más bien, lo que exige esa interpretación epistemológica es que respecto a lo que ya conocemos y hacemos, como a lo que vamos conociendo, debemos afrontarlo desde una unidad de sentido pedagógico consistente en que pensemos y actuemos tecnológicamente (Cf. Sarramona, 1990, 42-44).

Del mismo modo, cuando se indica acertadamente que la pedagogía ha dejado de ser una mera derivación de teorías o cosmovisiones filosóficas o antropológicas, no se está negando la importancia de ese factor como un elemento que posibilita la acción educativa. Lo que se cuestiona es que dirija el sentido pedagógico de la acción educativa en su totalidad, esto es, que se constituya en una metanorma que valide por sí misma la acción educativa emprendida (Cf. Castillejo, 1985; Castillejo y Colom, 1987, 24-25). Esto quiere decir, en definitiva, que las concepciones epistemológicas no se caracterizan necesariamente por los elementos que excluyen de la acción educativa, sino por el sentido o interpretación que pretenden establecer entre todos ellos.

En realidad, a través de las generalizaciones epistemológicas lo que tenemos son estructuras amplias de conocimiento por las que percibimos cuándo nos encontramos ante problemas que consideramos pedagógicos y cuándo estamos elaborando soluciones que identificamos como pedagógicas (Cf. Vázquez, 1984). Además, como hemos indicado, la construcción de esa estructura de conocimiento no surge desde una teoría sobre un elemento de la acción educativa, sino de la percepción original que tengamos sobre lo que son las funciones de la educación. Por tanto, mantener una concepción epistemológica de la educación es como mantener la concepción del conocimiento que se considera necesario para determinar y resolver las funciones de la educación.

Así, para los defensores de una concepción tecnológica de la educación, cumplir con las funciones de la misma no conlleva, por ejemplo, la exclusión de las cuestiones éticas de la educación; éstas actuarán como fines y regulaciones externas de la acción educativa. Ahora bien, mantendrán que los interrogantes y soluciones que nos proporciona la forma de conocimiento que denominamos tecnología, son 
las formas correctas de preguntar y decidir sobre las funciones de la educación. El conocimiento tecnológico será lo propio de la acción educativa. Mientras que para los que mantienen una concepción de la educación como práctica moral, son los interrogantes y las soluciones éticas las que realmente nos hacen cumplir con las principales funciones de la educación (cf. Elliot, 1986, 242-250, 257-259; Tom, R. A., 1984, 95-97). Lo ético sería aquí una condición interna de la acción educativa, mientras que lo tecnológico sería una regulación externa.

Aunque el discurso sobre los diferentes elementos de la acción educativa siga elaborándose, su amplitud y ulterior referencia depende del modo en que se consideren las generalizaciones epistemológicas dominantes. Los temas de investigación que se derivan de estos hechos son, principalmente, dos. Por un lado, debatir que concepción epistemológica concreta es la más adecuada para resolver y plantear acertadamente las funciones de la educación. También forma parte de esta investigación el estudio de lo que se va a considerar «pertinente pedagógicamente» en los nuevos hechos, teorías o propuestas provinientes de otros campos del conocimiento. Por otra parte, surge un problema más general, que condiciona incluso las discusiones referidas a la primera cuestión. Se trataría de analizar las condiciones que configuran cualquier concepción epistemológica en pedagogía, en la medida que se pretende crear un cuerpo de teoría con carácter de validación autónoma.

Actualmente, se tiende a considerar que la Teoría de la Educación ha pasado por tres etapas: Teoría de la Educación como teoría filosófica; Teoría de la Educación como teoría aplicada y, por último, Teoría de la Educación como teoría científico-tecnológica. El debate surge cuando no se acepta hoy como conocimiento «educativo» el proporcionado por el cumplimiento de las exigencias de investigación de cada tendencia, sino sólo el proveniente de la última de las señaladas, que defiende el carácter tecnológico de la actividad educativa. En la misma medida que nosotros aceptamos que cada corriente representa un tipo de conocimiento «educativo» y no sólo un momento histórico del estado de la investigación pedagógica, no podemos desentendernos de las consecuencias que para el conocimiento pedagógico tiene reconocer el porqué son válidas y necesarias esas formas de conocimiento.

\section{EL CONOCIMIENTO EDUCATIVO EN LA TEORÍA DE LA EDUCACIÓN ENTENDIDA COMO CONJUNTO DE TEORÍAS FILOSÓFICO-EDUCATIVAS}

En este enfoque, el principal problema de la tarea educativa es su justificación. El conocimiento de las razones de deseabilidad de los fines a los que ha de encaminarse la educación justifican la actividad educativa, pero en ningún caso dichos fines explican los mismos procesos que acontecen en la educación. De aquí, que podamos afirmar que, para esta concepción, la explicación del fenómeno educativo empieza y termina en la «explicación ulterior» (justificación) de los fines a los que atender. Por eso el conocimiento de la educación forma parte y adquiere sentido de ser en otro tipo de preocupaciones intelectuales, en un conocimiento «enhebrado en la reflexión antropológica general sin posibilidad de aislar un campo de referencias autónomo que diferenciara lo que hoy llamamos cultura, civilización, literatura o educación, pero descubriendo lo que pudiéramos llamar intencionalidad metafísica 
de la totalidad del proceso del vivir humano» (García Carrasco, 1984, XXVIII). Esto es: pensar en la educación es pensar en el producto ideal que ha de lograrse, en el hombre «educado», en los fines que correponden a su naturaleza.

Mejorar la educación (facilitar el acceso a ella) es mejorar la claridad del bien deseado, justificar su valor y su posición de fin en la vida humana. Esto, evidentemente, puede ser fácil o difícil (las más de las veces difícil), pero a esta valoración sobre la dificultad de acceder al conocimiento certero del fin, no se añade, en esta corriente, la dificultad propia de la transmisión; la facilidad o dificultad de ésta se atribuye a la complejidad específica del fin.

En consecuencia, saber sobre educación en esta concepción es conocer los fines de la vida deseables porque los fines de la educación se identifican con éstos (cf. Touriñan, 1987, 69-72). Intervenir en la educación, por otro lado, es ejercer una práctica moral en la rectitud de las decisiones que hagan vislumbrar realmente la deseabilidad de los fines filosóficamente conocidos.

\subsection{Posibilidades y límites de las teorías filosófico-educativas para el conocimiento de las finalidades de la educación.}

Lo más destacable de las críticas establecidas contra esta concepción es que aun cuando se anulase, acertadamente, la pretensión de conocer a la educación exclusivamente desde esta corriente, se seguiría manteniendo que el conocimiento que nos proporciona de la educación tiene poco valor pedagógico. Se admite su utilidad para la pedagogía, pero se niega que en sí mismo tenga valor pedagógico. Así dice Touriñán que «el valor pedagógico del conocimiento de la educación en la corriente marginal es francamente escaso» (p. 248). Nuestra intención, como se ha indicado, estriba en mostrar que el conocimiento que nos proporciona esta concepción es un conocimiento valioso y necesario para poder aspirar a cumplir con los requisitos de conocimiento de las otras corrientes. Obviamente, no es todo el conocimiento pedagógico; incluso no es el más específicamente pedagógico, pero, sin él no hay conocimiento pedagógico global.

\subsubsection{Los Fines deseables de la vida y los Fines educativos}

La crítica establecida contra la concepción de una Teoría de la Educación como derivación de teorías filosóficas, no mantiene explícitamente que todo conocimiento de los fines tenga poco valor pedagógico. Más bien, se afirma que el tipo de conocimiento que respecto a los fines mantiene esta corriente tiene poco valor pedagógico.

Esta matización es importante. Querer incrementar el conocimiento de la educación a través de las corrientes histórico-filosóficas, es una posibilidad equivocada y ya superada incluso desde la Filosofía de la Educación (Cf. Ibáñez-Martín, 1989, 412-413). Sin embargo, no tiene el mismo significado criticar para la educación el modelo de conocimiento de las corrientes filosóficas, que descalificar también para el conocimiento de la educación el estudio filosófico-educativo de los fines deseables de la vida.

Cuando se sitúan al mismo nivel ambos tipos de críticas es que está identificando el conocimiento de los fines deseables de la vida con el conocimiento histórico 
y sociológico de lo que impera socialmente. Hoy no puede mantenerse que el conocimiento de los fines generales del hombre sea sólo una adopción acrítica de corrientes filosóficas o ideológicas. Por otro lado, al establecer la distinción entre fines generales del hombre y fines educativos, lo que hay que precisar es que en esa diferenciación se está caracterizando al conocimiento pedagógico de esos fines en su ajuste técnico porque es una exigencia de la práctica educativa, pero no puede mantenerse que este ajuste sea sólo lo que delimita singularmente el conocimiento pedagógico de los fines.

El conocimiento de los fines se adquiere a través del conocimiento del hombre. El carácter de valor o de deseabilidad del fin surge de su correspondencia con el conocimiento de lo considerado como bueno para el hombre. Es cierto que lo bueno para el hombre en general no tiene porqué serlo para el hombre-educando en particular. También es verdad que lo bueno para el hombre-educando en particular no tiene porqué serlo de igual modo para el hombre en general. Las concretas circunstancias externas e internas del alumno pueden no hacer factible en un momento determinado la consecución del fin «objetivamente» deseable. De aquí, que sea cierto afirmar, como ya se indicó, que los fines deseables de la vida no se identifican sin más con los fines educativamente asequibles. Sin embargo, veamos con más detenimiento las limitaciones de estos hechos.

La concepción tecnológica de la educación defiende un saber pedagógico «sustantivo» (cf. Castillejo, 1985) que esté centrado en los procesos. En consecuencia, el conocimiento filosófico de los fines de la vida, de hecho, carece de utilidad pedagógica mientras no quede probado el ajuste técnico de esos fines. Los fines educativos son fines deseables, que, en una situación concreta, se considera recomendable su logro (asequibles al sujeto y a sus circunstancias). En definitiva, el saber educativo da la pertinencia precisa a los fines deseables, convirtiéndolos así en fines educativos. Ahora bien, en nuestra opinión, la argumentación anterior precisa las siguientes matizaciones:

* No existe realmente una separación nítida entre fines deseables de la vida y fines educativos. Detenerse en el ajuste técnico del fin (posibilidad y contenido de la intervención), supone en realidad permanecer en el mismo fin deseable, sometiéndole a prueba en su carácter de aconsejable y alcanzable por el alumno, en tanto que alumno.

* Del hecho de que en alguna ocasión quede probado pedagógicamente la no conveniencia de ocuparnos en un fin deseable, no puede deducirse un fin educativo mejor, sino tan sólo la necesidad de establecer las condiciones necesarias para poder posteriormente dirigimos a ese fin deseable.

De este modo, el ajuste técnico es una condición de aplicación del fin, —una de las muchas que puede tener-, pero no es una condición del conocimiento del fin como humanamente deseable. En la medida que permanecemos en el fin deseable para establecer su ajuste, la peculiaridad del conocimiento pedagógico y, por tanto, su valor, radicará tanto en la competencia técnica para adaptarlo, como en la reflexión teórica para justificarlo como fin formativo.

Admitir que podemos ir acumulando información científica y técnica sobre fines educativos, supone reconocer que lo que se retiene como valioso no son sólo los criterios técnicos de ajuste, sino los criterios de deseabilidad. El modo en que se 
concibe el carácter autónomo o sustantivo del saber pedagógico a través de la concepción tecnológica, sugiere que el valor educativo de los fines reside en establecer su adaptación al sujeto. Así, se mantiene que la tarea específica de la Pedagogía no estriba en reflexionar sobre la valiosidad de los fines, sino sobre su adaptabilidad y ajuste a las diversas dimensiones de la educación (cf. Castillejo, 1985, 51). Sin embargo, no es posible acumular información sobre criterios de ajuste si primeramente no se observa la necesidad de establecer esa adaptación por el grado de valor formativo que se divisa en esos fines, esto es, por ser un fin deseable de la vida. Además, cono intentaremos mostrar en la segunda parte de este trabajo, en la educación los sujetos no reciben estrictamente cambios o ajustes externos, sino que asumen para sí cambios, esto es, los criterios de lo que es valioso no son referencias externas a la dimensión productiva del profesor, sino que han de formar parte del mismo concepto de ajuste técnico.

Para mostrar con más detenimiento nuestros argumentos vamos a analizar los dos errores que creemos se mantienen en las posturas anteriores.

a) No es correcto identificar el conocimiento del ajuste técnico de los fines, con el conocimiento global de los fines educativos.

De la aplicación de la finalidad-del-conocimiento-pedagógico de la educación a algo (establecer o no su «carácter pedagógico»: alcanzable, pertinente, contextualizado, nivelado psicológicamente, secuencializado, etc.), sólo se puede concluir la presencia de esa finalidad-del-conocimiento-pedagógico en ese algo, pero en ningún caso es correcto concluir que ese algo ya es fin, objetivo o meta de la educación.

Si esto fuese así, nuestras investigaciones sobre educación y cualquier propuesta de finalidades no tendría más problemas, en orden a calificar a éstos de fines de la educación, que aplicarles la condición del conocimiento pedagógico (probar su carácter pedagógico: enseñarlos, saberlos situar en los diseños instructivos, relacionarlos con los métodos y las actividades, aprender a evaluarlos, etc.). De hecho, una de las razones por las cuales proliferan tantos y tan diversos fines de la educación -incluso contradictorios-, reside en la aplicación del razonamiento anterior.

Es cierto que los fines educativos, en tanto que objeto de instrucción, deben ser revisados para poder probar su «carácter pedagógico» en la forma indicada. No es cierto, sin embargo, que el conocimiento de los fines educativos, en tanto que fines y educativos, se resuelva sólo a partir del conocimiento de su accesibilidad en los procesos educativos. El que algo (recursos, procesos, métodos, etc.) me haga llegar a la meta que deseo, no otorga valor a la meta a la que me conduce.

No es lo mismo, pedagógicamente hablando, justificar el tiempo de dedicación a un nuevo contenido o fin de aprendizaje porque se ha establecido la posibilidad de incluirlo (carácter pedagógico), en una serie de métodos instructivos ya utilizados, porque se han conseguido delimitar unos conceptos específicos, porque se saben diseñar programaciones con todos sus elementos necesarios, etc., no es lo mismo, como decía, justificar o probar un nuevo fin y contenido instructivo porque se ha conseguido verificar su accesibilidad didáctica, que justificarlo y probarlo porque se sabe desvelar nítidamente «que se aprende tal o cual contenido para cubrir un sector relevante de la formación humana y no sólo para saber de él» (Castillejo, 1987, 13). 
Justificar que un fin deseable para la vida humana es también un fin adecuado de formación es crear conocimiento pedagógico, autónomo, valioso y no referido exclusivamente al ajuste técnico de ese fin.

No hay conocimiento «sustantivo» de la educación, ni tan siquiera pedagógico, cuando la única ocupación, desde los profesionales de la educación, es duplicar los argumentos filosóficos de valiosidad objetiva de un fin. El que esto históricamente se haya hecho, no invalida la necesidad de probar en la pedagogía, no el valor objetivo del fin, sino su virtualidad real para valorar la estructura personal del alumno en tanto que alumno. Pues bien, esta tarea no es todavía identificable con los ajustes técnicos necesarios para llevarlo a la práctica. Es cierto que al pensar en la virtualidad formativa de un fin o de un contenido me aproximo al proceso necesario para su accesibilidad operativa, pero no porque diseño técnico y valor formativo se identifiquen, sino porque el valor formativo de un fin es un conocimiento práctico y no especulativo. Entre el conocimiento general del valor de un fin y su planificación concreta en orden a su logro, hay un campo específico de validación pedagógica de carácter teórico. Por eso, «la reflexión educativa debe añadir a la preocupación filosófica por tratar de resolver del modo más adecuado ciertos problemas fundamentales, la preocupación por construir una imagen global y coherente que engarce todos los factores que intervienen en la formación de aquella faceta de la personalidad humana que en ese momento nos preocupa» (Puig y Martínez, 1989, 32).

Veamos seguidamente algunos hechos que pueden confirmar nuestros supuestos.

* Que se mantenga como fin educativo algo que la ciencia pedagógica todavía desconozca en su exacta regulación educativa. Por ejemplo, la alfabetización informática. Si lo mantiene es que, de algún modo, divisa el valor educativo que tiene para la formación del sujeto, aunque no pueda, por el momento, dar razón de ello recurriendo sólo a los procesos implicados en ese aprendizaje, es decir, al ajuste técnico preciso del fin pretendido (Cf. Vázquez, 1987, 209-210).

* Que se rechace la categoría de fin educativo a algo que la ciencia pedagógica sabe regular, probar, pronosticar, etc. por ejemplo, el aprendizaje mecánico-acumulativo. De la capacidad funcional de operar internamente que tiene la ciencia pedagógica, no pueden derivarse los fines educativos. Si la Pedagogía puede rechazar con fundamento el fin educativo del aprendizaje mecánico-acumulativo, aun cuando tenga los suficientes elementos para realizarlo correctamente, es que tiene que poseer como conocimiento acumulado otras condiciones que determinen los fines educativos, las cuales no podrán referirse solamente al carácter alcanzable de esos fines.

* Que se mantenga como fin deseable de la vida y como fines educativos derivados de éste, objetivos escasamente alcanzables por la mayoría de la población. Por ejemplo, el altruismo y su fin educativo el pensamiento moral en el estadio 6 del nivel posconvencional de Kohlberg (cf. Fierro, 1986, p. 116).

b) No es correcto considerar sólo con valor pedagógico al conocimiento que incrementa nuestro saber sobre los procesos educativos.

Los procesos educativos constituyen lo que se considera que es el componente teórico y práctico específicamente pedagógico. Su denominación y objeto de estu- 
dio se vincula con las corrientes de pensamiento actualmente más fructíferas: la psicología cognitiva y el enfoque del procesamiento de la información. Por otro lado, recogen como supuesto base la comprobación del carácter secundario de los contenidos o, mejor dicho, la dependencia de estos de los procesos y operaciones con los que se hace trabajar al alumno, en orden a obtener niveles óptimos de aprendizaje significativo. Finalmente, mantenerse o centrar la atención en los procesos es la consecuencia y el requisito lógico de concebir el conocimiento de la educación como conocimiento científico-tecnológico.

A pesar de todo, consideramos que la causa del error de concebir la especificidad educativa de los fines, sobre todo, a su ajuste técnico, reside en otorgar sólo valor pedagógico al conocimiento centrado en los procesos educativos. Esto es, a nuestro parecer, arriesgado e inexacto. Por un lado, creemos que es arriesgado porque, como hemos visto, existe la posibilidad - nada despreciable - de reconocer valor educativo en algo al tiempo que todavía desconocemos los procesos implicados en su consecución. A las pruebas ya señaladas hay que añadir otras:

* A la vista de unos resultados tendremos que reconocer valor educativo a la educación prestada por unos padres aunque no puedan darnos razones fundadas de los procesos que han utilizado. Es cierto que un profesional de la educación debe saber explicar por qué hay valor educativo en la acción de los padres, esto es, saber ver lo que ellos no ven; pero lo importante es que esos padres han producido unos efectos valiosos sin reconocer las variables relevantes y su orden. Esto no sucede, de igual modo, en otras ciencias, ni con otras prácticas.

* La posibilidad, nada remota tampoco, de obtener efectos no previstos en la aplicación de determinados procesos reconociendo al mismo tiempo que son efectos educativos valiosos, nos muestran la necesidad de que el profesional posea un margen de referencia sobre la valiosidad educativa más amplio que el derivado de los procesos implicados en una acción educativa concreta. Es arriesgado en educación dar por válido y valioso sólo lo previsto.

Por otro lado, otorgar valor pedagógico sólo al conocimiento que incrementa nuestro saber sobre los procesos educativos, es una afirmación inexacta porque no se ajusta al hecho educativo:

* Un problema es el modo en que tenemos que pensar y actuar en la educación para construir un conocimiento del mismo que sea pedagógico; y otro asunto es considerar, erróneamente, que con tal que nos centremos en los procesos el conocimiento ya es pedagógico. Identificar ambos planteamientos limita la búsqueda de fuentes de conocimiento valiosas para la educación. Así, conviene reconocer que no todo enfoque de los procesos es conocimiento pedagógico (puede ser psicológico, biológico, etc), por lo que el concepto de valor pedagógico debe originarse en referencias más amplias, siendo los procesos un elemento o momento de aplicación de ese valor pedagógico. Sólo así es posible reconocer valor pedagógico a estudios que se centran en destacar el valor formativo de ciertos fines deseables de la vida. 


\section{EL CONOCIMIENTO EDUCATIVO EN LA TEORÍA DE LA EDUCACIÓN ENTENDIDA COMO TEORÍAS EDUCATIVAS APLICADAS}

En términos generales - puesto que como veremos hay varios modos de entender el sentido del término «teorías aplicadas»-, esta generalización epistemológica fundamenta el conocimiento científicamente posible de la educación en el conocimiento que nos proporcionan las ciencias generadoras o ciencias de la educación. La «sustantividad» del conocimiento pedagógico queda cuestionada al considerar que los principios de intervención educativa no son un campo independiente de estudio, sino que se originan en los mismos principios descriptivos y valorativos que nos proporcionan las ciencias de la educación.

En esta acepción epistemológica se diferencian dos maneras de entender esas teorías de las disciplinas generadoras de las que surgirán las reglas de actuación o intervención educativa (Carr, 1990, 78-81). Por un lado, como teorías prácticas de la educación cuya utilización ha de referirse a aplicaciones técnicas. Por otra parte, otros defensores o partidarios de esta corriente mantienen que esas teorías prácticas han de referirse a aplicaciones éticas o deliberativas y no sólo técnicas.

\subsection{Posibilidades y límites de las teorías aplicadas para el conocimiento de las finalidades de la educación}

Se ha afirmado que «la tesis de la subalternación debe rechazarse, no en lo que tiene de fecunda para la investigación educativa, sino en lo que tiene de paralizadora de otras vías de investigación. Dependencia disciplinar no es lo mismo que subalternación. En otras palabras, la tesis de la subalternación no necesita revisión, lo que necesita es suplementación; sus argumentos son coherentes con una concepción de disciplina subalternada; nuestras críticas son pertinentes en la misma medida que hay significación intrínseca en los términos educacionales y no les prestamos la atención debida desde la concepción subalternada del conocimiento de la educación» (Cf. Touriñán, 1987, 33).

Nuestro propósito estriba en mostrar las razones por las que esta corriente tiene interés para la investigación educativa. Consideramos, en primer lugar, que la acertada y necesaria distinción entre dependencia disciplinar y sustantividad del conocimiento pedagógico, no puede basarse en una valoración diferente del conocimiento pedagógico generado en ambos modos de trabajar. Se puede mantener esa distinción entre dependencia y autonomía, equiparando al mismo tiempo el valor pedagógico de sus propuestas, si comprendemos que la aplicación es más un momento de investigación de la Pedagogía - y en muchos casos el único- que un paradigma de investigación. Por eso, en segundo lugar, el sentido de esta corriente de conocimiento no se alcanza a comprender si se repara sólo en que constituye un momento histórico de aceptación de paradigmas. La fecundidad de sus postulados no se debe exclusivamente a este planteamiento, esto es, no producen conocimiento porque nos situemos en una perspectiva de la Pedagogía como ciencia aplicada, sino porque en la mayoría de los problemas pedagógicos hay que partir de esa perspectiva metodológica de la aplicación para poder crear la necesaria «significación intrínseca» de los términos educativos, y realizar así una correcta validez pedagógica en términos 
de intervención. En tercer lugar, y refiriéndonos ya a los fines educativos, la habitual aplicación de otros datos y teorías al problema de los fines no es una limitación actual de nuestro conocimiento pedagógico, ni un indicador que descalifique su conocimiento como «cuestión no pedagógica», sino el segundo momento de investigación, siendo el primero el conocimiento filosófico-educativo de los fines deseables de la vida que veíamos más arriba.

Consideramos, por tanto, que hay que mantener los supuestos más importantes de esta corriente de conocimiento pedagógico por las siguientes razones:

a) Cuando se defiende — acertadamente - la necesidad de referirnos a un ámbito de validez pedagógica que sea autónomo respecto de otras disciplinas, suele darse la impresión de que ya está hecho del todo, que sólo tenemos que referirnos a él para probar qué es y qué no es útil y valioso en la educación. Esto no es así. Más bien, el dato importante es que se va construyendo paulatinamente. De este modo, al no estar determinado del todo cómo dar validez pedagógica, al no tener resuelto definitivamente los criterios de significación intrínseca de los datos y de las teorías educativas, no es prudente descalificar las aplicaciones teóricas de otras ciencias al campo educativo.

Conviene distinguir la necesidad epistemológica de ese ámbito autónomo de validación, de lo que puede llegar a ser valioso pedagógicamente, aunque forme parte de otras disciplinas. Sin esa diferencia llegaremos a entender a las ciencias auxiliares de la educación y a las ciencias fundamentales como ciencias de la pedagogía y no de la Educación (Filosofía de la Pedagogía, Psicología de la Pedagogía, Teoría de la Pedagogía, etc.). Esta posibilidad es aún mayor cuando se identifica la autonomía del saber pedagógico - una aspiración socialmente legítima y epistemológicamente necesaria- con el conocimiento pedagógico mismo.

b) Aunque fuese posible exigir a todas las finalidades de la educación, el requisito de que elaborasen una significación intrínseca - pedagógicamente sustantivade sus términos y teorías, pudiendo criticar, por tanto, el que permanecieran en meros saberes de aplicación de otras ciencias, la deseable pluralidad que hay respecto a los fines educativos seguiría sin desaparecer porque nadie admitiría como fin valioso aquel que mejor pudiese ser reinterpretado desde los principios de la pedagogía, sino el que considerásemos como realmente valioso para las nuevas generaciones desde todos los puntos de vista.

c) La participación puede ser un fin educativo, pero optar por no fomentar la participación en una situación concreta puede ser la decisión que mejor represente el valor educativo que deseamos que asuman los educandos. Esta diferencia demuestra la existencia de un ámbito de validación autónomo desde la pedagogía. Sin embargo, a pesar de la constatación evidente de este hecho sigue sin estar claro cómo podemos alcanzar ese ámbito de validación autónomo, toda vez, que lo que se constata también en el ejemplo es el carácter irremediablemente contingente, aplicativo y deliberativo de las situaciones educativas (cf. Kninght y Smith, 1989, 437; Egan, 1988, 71,76), esto es: lo más opuesto a la aspiración de una significación intrínseca de las finalidades y de los valores educativos.

Tal vez, la explicación radique en que cuando detectamos indicios de una ciencia pedagógica sustantiva (como en el caso de la participación), lo que realmente 
tengamos sea, de nuevo, la comprobación de la especificidad de lo educativo en cada uno de los sujetos, es decir, que lo sustantivo y autónomo es el modo en que el sujeto asume su educación a través de una práctica muy singular, y no necesariamente tiene que serlo el conocimiento estratégico o productivo que utiliza el educador.

d) Es conveniente diferenciar dos aspectos muy relacionados pero diferentes. La Pedagogía es la ciencia de la educación, pero la fuente de conocimientos de datos y situaciones susceptibles de educar es el propio sujeto en relación consigo mismo y con la realidad cultural que le rodea. El deseable rigor científico al que debe aspirar la pedagogía, no debe suponer la minusvaloración de un dato radicalmente importante: lo que un hombre hace o decide hacer para educarse a lo largo de su vida, no es una decisión científica, ni técnica sobre su formación, sino unas, quizás, simples deliberaciones entre deseos, gustos, ideas, actitudes, etc. El criterio de valor pedagógico no puede residir, por tanto, sólo en el cumplimiento de directrices epistemológicas internas a la Pedagogía, sino aspirar a aceptar múltiples vías de comprensión de lo educativo (conocimiento aplicado) independientemente de que no siempre podamos alejarnos de la dependencia disciplinar o de que no podamos analizarlas desde el rigor científico dominante.

En último término, deberíamos diferenciar en la epistemología pedagogía lo que procuró deslindar Bollnow $(1970,33)$ para una Filosofía del Conocimiento. Por un lado, hemos de desarrollar una Teoría del conocimiento pedagógico que nos asegure la certeza de los saberes establecidos; por otro, debemos aspirar a comprender al hombre y a todas sus situaciones educativas desde el conocimiento pedagógico y desde cualquier otro conocimiento.

e) El conocimiento educativo de algo, no perteneciente originariamente al campo de la educación se logra siempre, en primer lugar, a través de los supuestos-base de esta corriente de conocimiento, según los cuales, utilizamos la educación como campo de aplicación de saberes o cuestiones ni directa ni necesariamente educativas todavía. El conocimiento aplicado para la educación, cuando el objeto de investigación no es directamente de la educación (tal como ocurre ni más ni menos que con todas las propuestas de finalidades educativas), no debe transformarse inicialmente en conocimiento autónomo mediante la aplicación de las exigencias de esta corriente, porque la finalidad de nuestra búsqueda no es sólo el conocimiento de intervención educativa (el conocimiento de la educación en la corriente autónoma), sino el conocimiento de esos fines deseables en la educación, en el «funcionamiento» teórico y práctico de la misma.

El conocimiento de los fines educativos no puede consistir sólo en un saber de intervención, porque en la medida que se reconozca su importancia para estructurar el sentido de la acción educativa, se tiene que admitir necesariamente un conocimiento contextualizado de esos fines. Un saber tan generalizado de los mismos que nos descubra mediante aplicación en lo que ya sabemos o suponemos como cierto, qué incidencias tiene en la educación como realidad social y como aspiración personal de los ciudadanos (Cf. Keeves, 1988, 28). Esto es así salvo que equivocadamente se mantenga que la educación o el conocimiento que es valioso para la educación es el que se dirige sólo a analizar la relación técnica entre medios y fines. 
Todo lo dicho implica que la construcción de teorías aplicadas para la educación no ha de entenderse siempre como un modelo secundario de conocimiento, cuya existencia adquiere sentido en la medida que lo transformemos lo más pronto posible en conocimiento autónomo de la educación. Las teorías aplicadas proponen modalidades de acceder al conocimiento de la educación o resaltan dimensiones de éste, que no contempla suficientemente la corriente autónoma de conocimiento. Ser conscientes de este hecho es lo que realmente nos puede permitir aspirar a una ciencia de la educación autónoma, interdisciplinar y abierta. Como práctica social y personal de aprendizaje, la educación no se llega a conocer por permanecer en lo que se hace o se ha de hacer en las instituciones formales de enseñanza, sino por partir de un marco de validación de tendencias más amplio: el que se origina en la sociedad y el que van adoptando los sujetos. El supuesto de la aplicación disciplinar de conocimiento a la educación es así imprescindible para pensar una educación global y sujeta a la realidad.

Todas estas virtualidades de la investigación que muestran los supuestos-base del conocimiento aplicado en la educación han tenido, de hecho, resultados fundamentales para el conocimiento de la teoría y de la práctica educativa. Un caso especialmente significativo lo constituye, en nuestro contexto, el amplio y acertado desarrollo teórico de la Teoría de la Educación como conocimiento científico-tecnológico.

La defensa actualmente mayoritaria de esa afirmación (cf. Escámez, 1988, 1314) no tiene una significación intrínseca originada en un ámbito de validez pedagógico específico y autónomo. Mantener que el conocimiento de la educación es conocimiento científico-tecnológico es la consecuencia de aplicar o de probar lo que se conocía de la educación o sobre la educación en el conocimiento epistemológico en vigor. Este establece el qué es y cómo se desarrolla lo científico y lo tecnológico, qué tienen en común, en qué se diferencian, a qué se aplica y qué pruebas de validez tiene. Por otro lado, se recoge lo que se sabe sobre educación, en orden a aplicar en ésta, aquellos requisitos; unas veces, comprobando que los cumple y, otras, haciendo que los satisfaga. En cualquier caso no ha surgido esa «innovación» desde un ámbito específicamente pedagógico, ni en sentido estricto es mantenible que se haya validado pedagógicamente a través de una significación instrínseca. Simplemente se ha tenido el enorme acierto de ajustar, aplicar o probar el conocimiento epistemológico sobre tecnología en cuestiones educativas.

Bien podríamos decir que la defensa de un ámbito de validez pedagógico con carácter «sustantivo» en sus términos y en sus teorías pedagógicas (tecnológico), es una reivindicación posible por las consecuencias teóricas obtenidas tras el conocimiento aplicado de los saberes epistemológicos en la educación. 


\section{BIBLIOGRAFÍA}

Bollnow, O. (1970): Introducción a la Filosofía del Conocimiento Amarrortu, Buenos Aires.

CARR, W. (1990): Hacia una ciencia crítica de la educación. Laetres, Barcelona.

CASTAÑE, J. (1990): «Tecnología de finalidades pedagógicas». Revista Complutense de Educación, 1:1. pp. 109-120.

CASTILlEJO, J. L. (1985): «Sugerencias para una teoría de la ciencia pedagógica», en Varios. Conceptos y propuestas (II), pp. 45-56. Nau Llibres. Valencia.

CASTILLEJO, J. L. (1987): «Investigación y acción educativa: Un modelo de integración de la teoría y la praxis», en Varios: Investigación educativa y práctica escolar, pp. 9-31. Santillana, Madrid.

CASTILlejo, J. L. y COLOM, A. J. (1987): Pedagogía Sistémica. CEAC. Barcelona.

DIKMEYER, N. (1989): «Metaphor, model, and theory in education research». Teachers college Record, 91:2, winter, pp. 151-160.

EGAN, K. (1988): «The analytic and the arbitrary in educational reearch». Canadian Journal of Education, 13:1, pp. 69-82.

ELLIOT, J. (1986): «Autoevaluación, desarrollo profesional y responsabilidad,» en GALTON, M. y MOON, B.: Cambiar la escuela, cambiar el curriculum, pp. 237-259. Martínez Roca, Barcelona.

ESCÁMEZ, J. (1988): «La teoría pedagógica y el progreso educativo», en S.E.P. La calidad de los centros educativos. S.E.P., Alicante.

FIERRO, A. (1986): Desarrollo social de la personalidad en la adolescencia, en CARRETERO, M.; PAlACIOS, J. Y MARCHESI, A. (Comps.): Psicología Evolutiva 3, pp. 95-142. Madrid, Alianza.

GARCÍA CARRASCO, J. (1984) (coord.). Teoría de la Educación. Diccionario de Ciencias de la Educación. Anaya. Madrid.

IBAÑEZ, MARTÍN, J. A. (1989): «El concepto y las funciones de una Filosofía de la educación a la altura de nuestro tiempo», en Varios: Filosofía de la Educación Hoy, pp. 409. 419. Dykinson, Madrid.

KEEVES, J. (1988): «The unity of educational research.» Interchange, 19:1, Spring, pp. 14-30.

KNIGHT, P. Y SMITH, L (1989): «In search of good practice». Journal of Curriculum Studies, 21:5, pp. 427-440.

LARRoSA, J. (1990): El trabajo epistemológico en Pedagogía. PPU, Barcelona.

MEdINA LIBERTY, A. (1989): Psicología y epistemología. Trillas, México.

NAGEL, E. (1974): La estructura de la ciencia. Paidós, Buenos Aires.

PUIG ROVIRA, J. M. Y MARTíneZ MARTÍN, M. (1989): Educación moral y democracia. Laertes, Barcelona.

SARRAMONA, J. (1990): Tecnología educativa (una valoración crítica). CEAC, Barcelona.

SOCKETT, H. (1989): A moral epistiemology of practice? Cambridge Journal of Education, 19:1, pp. 33-39.

SOLTIS, J. F. (1988): Dewey and Thorndike: the persistence of paradigms in educational scholarship. Canadian Journal of Education, 13:1. pp. 39-51.

TOM, R. A. (1984): Teaching as moral craft. Longman, New York.

TOURIÑAN, J. M. (1987): Teoría de la Educación. Anaya, Madrid.

TOURIÑAN, J. M. (1989): «Las finalidades de la educación: Análisis teórico», en ESTEVE, J. M. (ed.): Objetivos y Contenidos de la Educación para los años noventa, pp. 15-34. Universidad de Málaga. 
VÁzQuEZ, G. (1984): La Pedagogía General, ¿Una teoría general de la Educación? Problemática actual y perspectivas de futuro, en Cincuentenario de los Estudios Universitarios de Pedagogía, pp. 13-33. Universidad Complutense, Madrid.

VAZQUEZ, G. (ed.) (1987): Conclusiones y Recomendaciones, en Educar para el siglo XXI., pp. 209-214. Fundesco, Madrid. 